\title{
Aplicações de indicadores como estratégia de gerenciamento do uso e custo dos antimicrobianos em um hospital universitário
}

\author{
Applications of indicators as a strategy for the management of the use and cost of antimicrobials in \\ a university hospital
}

Aplicaciones de indicadores como estrategia de gestión del uso y coste de antimicrobianos en un hospital universitario

\section{Resumo}

O aumento no consumo dos antimicrobianos tem resultado em elevados custos para a saúde pública, além de impactar no aumento da resistência microbiana como um problema mundial. Este estudo teve como objetivo avaliar o consumo e o impacto econômico dos antimicrobianos em um Hospital Universitário, por meio da análise do uso e custos, no período de 2015 a 2017. Trata-se de um estudo longitudinal, analítico com coleta de dados retrospectiva. Calculou-se o seu consumo através dos indicadores preconizados pela Organização Mundial da Saúde e por último, analisou-se o custo do consumo dos antimicrobianos através dos valores unitários em reais (R\$). Para a análise estatística foi utilizado o teste t e a significância estatística com intervalo de confiança 95\%. Por meio do cálculo da dose diária definida, os resultados mostraram diminuição no consumo no primeiro ano, seguido de aumento do consumo entre os anos, em especial, as classes dos carbapenêmicos, penicilinas, cefalosporinas e glicopeptídeos. Enquanto, o tempo de tratamento mensurado apresentou diminuição durante o período em estudo. Com relação ao custo, houve redução entre 2015 e 2016 e logo após um aumento entre 2016 e 2017. Conclui-se que os métodos de análise de consumo, são ferramentas que norteiam o gerenciamento do uso racional dos antimicrobianos e contribuem para a diminuição nos custos no ambiente hospitalar.

Palavras-chave: Antimicrobianos; Uso de medicamentos; Custos com medicamentos.

\begin{abstract}
The increase in the consumption of antimicrobials has resulted in high costs for public health, in addition to impacting the increase in microbial resistance as a worldwide problem. This study aimed to assess the consumption and economic impact of antimicrobials in a University Hospital, through the analysis of use and costs, in the period from 2015 to 2017. It is a longitudinal, analytical study with retrospective data collection. Their consumption was calculated using the indicators recommended by the World Health Organization and finally, the cost of consumption of antimicrobials was analyzed using the unit values in reais ( $\mathrm{R}$ \$). For the statistical analysis, the $\mathrm{t}$ test and statistical significance were used with a $95 \%$ confidence interval. By calculating the defined daily dose, the results showed a decrease in consumption in the first year, followed by an increase in consumption between years, especially the classes of carbapenems, penicillins, cephalosporins and glycopeptides. Meanwhile, the measured treatment time decreased during the study period. With regard to cost, there was a reduction between 2015 and 2016 and shortly after an increase between 2016 and 2017. It is concluded that the methods of consumption analysis are tools that guide the management of the rational use of antimicrobials and contribute to the decrease in costs in the hospital environment.
\end{abstract}

Keywords: Antimicrobial agents; Drug utilization; Drug costs. 


\begin{abstract}
Resumen
El aumento en el consumo de antimicrobianos ha resultado en altos costos para la salud pública, además de impactar en el aumento de la resistencia microbiana como problema mundial. Este estudio tuvo como objetivo evaluar el consumo y el impacto económico de los antimicrobianos en un Hospital Universitario, a través del análisis de uso y costos, en el período de 2015 a 2017. Se trata de un estudio longitudinal, analítico con recolección de datos retrospectiva. Su consumo se calculó utilizando los indicadores recomendados por la Organización Mundial de la Salud y finalmente, se analizó el costo del consumo de antimicrobianos utilizando los valores unitarios en reales ( $R$ \$). Para el análisis estadístico se utilizó la prueba t y la significación estadística con un intervalo de confianza del 95\%. Al calcular la dosis diaria definida, los resultados mostraron una disminución en el consumo en el primer año, seguido de un aumento en el consumo entre años, especialmente las clases de carbapenémicos, penicilinas, cefalosporinas y glicopéptidos. Mientras tanto, el tiempo de tratamiento medido disminuyó durante el período de estudio. En cuanto al costo, hubo una reducción entre 2015 y 2016 y poco después un aumento entre 2016 y 2017. Se concluye que los métodos de análisis de consumo son herramientas que orientan la gestión del uso racional de antimicrobianos y contribuyen a la disminución de costes en el entorno hospitalario.
\end{abstract}

Palabras clave: Antimicrobianos; Uso de medicamentos; Costos de medicamentos.

\title{
1. Introdução
}

A descoberta dos agentes antimicrobianos foi um marco para a medicina, revolucionando a assistência médica do diagnóstico ao tratamento de infecção bacteriana. No entanto, paralelamente ao benefício da utilização dos medicamentos a descoberta, estão o seu uso inadequado, que contribuíram para o crescente aumento da resistência bacteriana, com consequente aumento dos custos hospitalares segundo (Lobo et al., 2017).

O aumento da resistência bacteriana gerando uma redução na eficácia dos antimicrobianos, faz com que o tratamento dos pacientes seja difícil, caro ou até mesmo inexistente (Grosbelli et al., 2020). Neste caso, o aumento no consumo dos antimicrobianos tem resultado em elevados custos para saúde pública, pois estas despesas podem alcançar mais de $50 \%$ dos gastos gerais com medicamentos em um hospital. Deste modo, ainda estão entre os grupos farmacológicos mais prescritos, o que demonstra o aumento do seu consumo ao longo do tempo (Santos et al., 2021).

Em vista disso, são utilizadas estratégias na busca de melhores resultados para tentar controlar o consumo dos antimicrobianos na dispensação em hospitais. A restrição do seu uso é um método amplamente utilizado para o controle da prescrição e consiste na utilização de antimicrobianos somente após a avaliação e liberação pela Comissão de Infecção Hospitalar (CCIH), Brasil (2017). Em meio a este cenário o monitoramento do consumo desses antimicrobianos tem se tornado um instrumento de grande interesse e tem merecido uma atenção particular nos últimos anos Grosbelli et al., (2020).

Neste sentido, a Organização Mundial de Saúde (OMS) preconiza métodos para mensurar o consumo de antimicrobianos como ferramentas para análise de utilização de medicamentos através da metodologia ATC/DDD (Anatomical Therapeutic Chemical / Defined Daily Doses) e DOT (Days of Therapy) que permite dimensionar se o uso destes fármacos está aumentando ou regredindo, World Health Organization - WHO. (2012). Bem como a Gerência de Tecnologia em Serviços de Saúde (GGTES/ANVISA) por meio da nota técnica $n^{\circ}$ 05/2017 estabeleceu a realização do monitoramento nacional do consumo de antimicrobianos, Brasil (2017).

Assim, o estudo teve como objetivo geral avaliar o consumo e o impacto econômico dos antimicrobianos em um Hospital Universitário, analisando o uso e custos, no período de 2015 a 2017.

\section{Metodologia}

O estudo foi realizado no Hospital Universitário da Universidade Federal do Piauí, que conta com aproximadamente 190 leitos de internação (175 na Unidade de Internação e 15 na Unidade de Terapia Intensiva), no período de 2015 a 2017. 
Trata-se de um estudo longitudinal, quantitativo analítico com coleta de dados retrospectiva, na qual os dados secundários foram coletados após parecer positivo do Comitê de Ética. As variáveis estudadas foram: Indicadores DDD e DOT e custo dos antimicrobianos.

A análise dos dados foi realizada em três etapas. Primeiramente, foram verificados os antimicrobianos padronizados no período em estudo. Assim, foram analisadas 13 classes de antimicrobianos: aminoglicosídeos, carbapenêmicos, cefalosporinas, glicilciclinas, glicopeptídeos, lincosamidas, macrolídeos, nitroimidazois, axazolidinonas, penicilinas, polimixinas e quinolonas. Em suas apresentações de via oral (VO) e parenteral.

Em seguida foram utilizados dados secundários contidos em uma tabela de controle de consumo de antimicrobianos padronizada pelo setor de Comissão de Controle de Infecção Hospitalar (CCIH) com informações sobre os antimicrobianos utilizados, dose, via de administração, posologia, Unidades de Internação, dias de tratamento dos antimicrobianos e dados de relatórios gerencias do Aplicativo de Gestão para Hospitais Universitários (AGHU).

De acordo com a metodologia preconizada (OMS), foram calculados o consumo dos indicadores Doses Diárias Definidas (DDDs) por 1000 pacientes-dias por meio do levantamento da quantidade consumida, em gramas, de cada medicamento, dividido pela DDD padrão segundo a versão 2016 do sistema ATC/DDD. Em seguida dividido pelo número de pacientes-dia e multiplicado por 1000, utilizando a seguinte fórmula:

$$
\mathrm{DDD}=\frac{\mathrm{A} / \mathrm{B}}{\mathrm{P}} \times 1.000
$$

Onde: $\mathrm{A}=$ Quantidade total de antimicrobiano utilizado expresso em gramas; $\mathrm{B}=$ Dose diária padrão estabelecida para o medicamento em gramas; $\mathrm{P}=$ Pacientes-dia no período observado.

Para o cálculo dos Dias de Tratamento (DOTs) por 1000 pacientes-dia foi realizado a partir da soma de dias de tratamento dos antimicrobianos dividido pelo número de pacientes-dia e multiplicado por 1000, como mostra a fórmula a seguir:

$$
\text { DOT }=\underset{\text { P }}{\mathrm{P} \_} \times 1.000
$$

Onde: $\mathrm{A}=$ Soma total de dias de uso do(s) respectivo(s) antimicrobiano(s) utilizado(s); $\mathrm{P}=$ Paciente-dia no período observado.

E por último, para a análise dos custos dos antimicrobianos, foi obtida uma média dos valores unitários em reais (R\$) mais recentes por grama adquiridos no hospital a partir do aplicativo AGHU através de notas de recebimento e transferidos para uma planilha no Microsoft Excel®. Posteriormente, foram calculados o seu custo e comparado ao consumo de antimicrobianos no período avaliado.

Os dados foram tabulados no Microsoft Excel e em seguida analisados utilizando o programa R Software (Licença Pública Geral GNU Geral da Software Foundation), especificamente o pacote R (versão 3.4.2). A pesquisa foi aprovada pela Comissão de Avaliação de Projeto (CAPP) n n 28/17 do Hospital Universitário da Universidade Federal do Piauí e após aprovação ao Comitê de Ética em Pesquisa (CEP) da referida instituição, por meio da Plataforma Brasil, sob o número de parecer 2.463.898. Foi solicitado o Termo de Consentimento Livre e Esclarecido (TCLE) e solicitada à autorização para uso de dados secundários por meio do Termo de Consentimento de Utilização de Dados (TCUD). 


\section{Resultados e Discussão}

Esta pesquisa foi estruturada a partir da análise do consumo de 13 classes de antimicrobianos, para as apresentações de via oral (VO) e parenteral, ao longo do período de estudo realizado em 2015, 2016 e 2017, conforme a relação de medicamentos padronizados em um Hospital Universitário. Assim, para mensurar o consumo desses antimicrobianos a OMS preconiza a utilização dos indicadores ATC/DDD (Anatomical Therapeutic Chemical / Defined Daily Doses) e DOT (Days of Therapy) por 1.000 pacientes dia, World Health Organization - Who (2012). Portanto, como expressa o gráfico 1, os indicadores expressos em DDD e DOT dos antimicrobianos do Hospital em estudo.

Gráfico 1 - Indicadores de consumo expressos em DDD e DOT de todos os antimicrobianos em estudo no período de 2015 a 2017 em um Hospital Universitário. Teresina-PI, 2018.

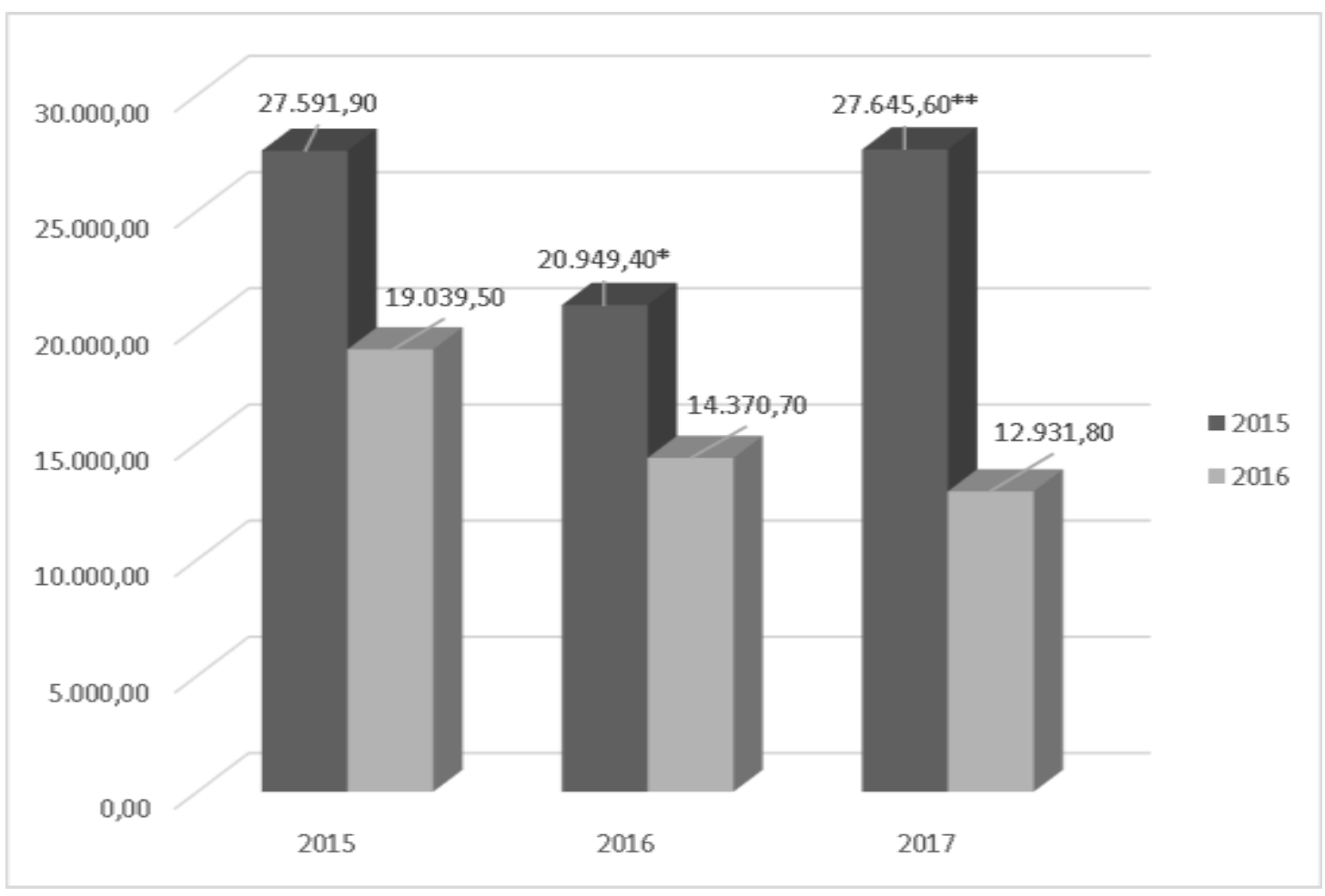

Legenda: A análise estatística foi baseada no teste $t$, que mostrou diferenças estatisticamente significativas para os indicadores: O DDD entre 2015 e 2016 apresentou uma diminuição estatisticamente significativa ( $\mathrm{p}=0,0035$ )*, já entre 2016 e 2017 observou um aumento estatisticamente significativa $(\mathrm{p}=0,04101)^{* *}$. Para o DOT teve uma diminuição estatisticamente significativa a cada ano $(\mathrm{p}<0,05)$ para todo o estudo.

Fonte: Autores.

Para o indicador de consumo expresso em DDD, apresentaram uma diminuição de estatisticamente significativa em 2015 para 2016 de 6.642,2, mas logo após um aumento no consumo de 2016 para 2017 de 6.696,2 por conta da contribuição das análises das receitas e a diminuição dos antibióticos. E para os valores expressos em DOT notou-se uma diminuição a cada ano analisado. Assim, observou-se que apesar do aumento no consumo dos antimicrobianos, o hospital teve uma diminuição nos dias de tratamento.

Corroborando estes dados com o estudo foi observada uma diminuição e logo após um aumento do consumo dos antimicrobianos. Entretanto, sugere-se que o alto consumo de antimicrobianos verificado deva-se a hábitos de prescrição, inadequada adesão a normas de uso apropriado, pouca experiência em programas de avaliação do uso de medicamentos e à complexidade dos procedimentos hospitalares realizados Momattin, et al. (2018). 
A seguir na Tabela 1, está exposta a relação de consumo por classes dos antimicrobianos padronizados no hospital universitário, expressos através dos indicadores DDD e DOT por 1000 pacientes-dia durante o período em estudo.

Tabela 1. Relação de consumo por classes de antimicrobianos em um Hospital Universitário, expressos em DDD/1000 pacientes-dia e DOT/1000 pacientes-dia no período de 2015, 2016 e 2017. Teresina-PI, 2018.

\begin{tabular}{|c|c|c|c|c|c|c|}
\hline \multirow{2}{*}{$\begin{array}{c}\text { Classes dos } \\
\text { antimicrobianos }\end{array}$} & \multicolumn{2}{|c|}{2015} & \multicolumn{2}{|c|}{2016} & \multicolumn{2}{|c|}{2017} \\
\hline & DDD & DOT & DDD & DOT & DDD & DOT \\
\hline Aminoglicosídeos & 477,9 & 496,4 & 495,8 & 444,7 & 328,2 & 218,6 \\
\hline Antifúngicos & 2030,7 & 1467,5 & 1822,9 & 1048,3 & 1497,8 & 900,5 \\
\hline Carbapenêmicos & 7073,6 & 3616,0 & 5391,3 & 3175,1 & 7740,1 & 2292,3 \\
\hline Cefalosporinas & 3790,6 & 2734,6 & 3293,9 & 2135,2 & 4324,8 & 2425,6 \\
\hline Glicilciclinas & 279,7 & 235,6 & 152,1 & 126,2 & 133,8 & 48,2 \\
\hline Glicopeptídeo & 2394,2 & 2496,6 & 2111,9 & 1846,6 & 2758,8 & 1494,2 \\
\hline Lincosamidas & 727,0 & 510,8 & 397,0 & 341,1 & 497,1 & 326,6 \\
\hline Macrolídeos & 527,1 & 327,3 & 300,6 & 189,9 & 364,3 & 179,2 \\
\hline Nitroimidazóis & 1640,4 & 1882,4 & 1281,8 & 1241,3 & 1606,9 & 1292,1 \\
\hline Oxazolidinonas & 437,9 & 370,7 & 126,2 & 127,0 & 545,5 & 324,3 \\
\hline Penicilinas & 4456,6 & 1764,9 & 3080,8 & 1407,2 & 4256,3 & 1615,8 \\
\hline Polimixinas & 588,5 & 1152,5 & 101,2 & 856,8 & 1535,6 & 583,1 \\
\hline Quinolonas & 3167,7 & 1984,3 & 2393,7 & 1431,1 & 2056,1 & 1231,2 \\
\hline TOTAL & $27.591,9$ & $19.039,5$ & $20.949,4$ & $14.370,7$ & $27.645,6$ & $12.931,8$ \\
\hline
\end{tabular}

Legenda: A análise estatística foi baseada no teste $t$, que se notou diferenças estatisticamente significativas para a relação dos indicadores DDD e DOT durante os anos 2015 ( $p=0,02634), 2016$ ( $p=0,008935)$ e 2017 ( $p=0,006029)$. Source: Authors.

A relação de consumo por classes dos antimicrobianos entre os indicadores DDD e DOT por 1000 pacientes-dia nos anos de 2015, 2016 e 2017 apresentaram diferenças estatisticamente significativas. Portanto, é importante ter um método que reconheça essas alterações no consumo, como o método DDD, e um método independente da dosagem, como o método DOT, para uma melhor mensuração do consumo de antimicrobianos dentro de um ambiente hospitalar, (Silva et al. 2020).

Embora os valores de DDD representem aproximadamente dias de terapia (DOT), existem grandes discrepâncias, principalmente devido à variedade de tamanhos de dose e frequências associadas a cada agente. Assim, o acompanhamento dos dias reais de tratamento a partir dos registros de administração é um método complementar, (Guillot, et al.,2014). Portanto, levando em consideração os dias de tratamento pode-se observar que houve uma diminuição nos valores do DOT para as classes de antimicrobianos no período de 2015 para 2016 e de 2016 para 2017. Assim, apesar do consumo em DDD terem aumentado nos últimos anos, os dias de tratamento dos antimicrobianos diminuíram. Do mesmo modo a diminuição nos dias de utilização dos antimicrobianos pode ser justificada pelas ações da CCIH em estabelecer prazos pequenos e até mesmo não autorizar o uso prolongado sem antes uma nova avaliação médica. Assim como também a não dispensação do antimicrobiano pelo farmacêutico após indeferimento da CCIH, (Vasconcelos, et al., 2015).

Estudos semelhantes, como o de Dubrovskaya, et al., (2017), que analisou no ano de 2015 o consumo de antimicrobianos na qual observou que o valor do DOT diminuiu. Assim como também Siegfried, et al., (2017), que com a presença dos residentes de farmácia ao longo de um período de doses meses (2014-2015) comparada aos 12 meses anteriores obteve uma diminuição significativa no DOT. Contudo, os dois estudos mostraram que com a presença do farmacêutico por meio de intervenções e análise dos formulários pela CCIH, são alternativas que podem melhorar a monitorização da utilização 
dos antimicrobianos reduzindo os dias de terapia. Desta forma, o hospital em estudo já realiza essas alternativas, podendo assim, ter sido uma das justificativas para a diminuição do DOT no período analisado.

Em relação aos valores de DDD, como mostra a tabela 1, observou-se que o consumo entre as classes de antimicrobianos que apresentaram uma diminuição foram os grupos dos carbapenêmicos, penicilinas, cefalosporinas e glicopeptídeos e logo após aumento no seu consumo. Já para os grupos antifúngicos, glicilciclinas e quinolonas tiveram uma diminuição em seu consumo a cada ano estudado.

Com ênfase nas três classes mais consumidas, o grupo que apresentou maior consumo durante o período em estudo foram os carbapenêmicos com uma queda de 2015 (7073,6 DDD/1000 pacientes-dia) a 2016 (5391,3 DDD/1000 pacientes-dia) e aumento para 2017 (7740,1 DDD/1000 pacientes-dia). Entretanto, por serem um poderoso grupo de antimicrobianos de amplo espectro e em muitos casos uma das últimas defesas efetivas contra infecções causadas por bactérias multirresistentes, como algumas cepas de Klebsiella pneumoniae e Escherichia coli, são considerados dados preocupantes para o hospital, pois, uma vez que há o aumento no consumo, consequentemente podem aumentar o número de cepas resistentes aos antimicrobianos, Wushouer et al., (2017).

Em seguida, o grupo das penicilinas foi à segunda classe de antimicrobianos que demonstraram altos valores do DDD durante os anos de 2015 (4456,6 DDD/1000 pacientes-dia), 2016 (3080,8 DDD/1000 pacientes-dia) e 2017 (4256,3 DDD/1000 pacientes-dia). Assim, mostraram que embora a diminuição e o aumento do consumo, mantiveram-se estáveis no período em estudo.

E a terceira classe de antimicrobianos que apresentaram maior consumo em DDD foi o grupo das cefalosporinas que demonstrou uma diminuição e logo após aumento nos períodos 3.790,6 DDD/1000 pacientes-dia em 2015, 3.293,9 DDD/1000 pacientes-dia em 2016 e 4.324,8 DDD/1000 pacientes-dia em 2017. Para isso, alguns estudos relatam que esse aumento pode ser influenciado pelo grande número de tratamentos profiláticos em pacientes cirúrgicos, tendo em vista que o hospital em estudo aumentou o número de cirurgias entre o ano 2016 (4.745 cirurgias) a 2017 (6.079 cirurgias), Wushouer, et al. (2014) e Rodrigues, et al., (2010).

Do mesmo modo, corroborando com os dados encontrados neste estudo, Bitterman, et al. (2016), mostrou que as classes mais consumidas foram das cefalosporinas, seguida de carbapenêmicos e glicopeptídeos. Enquanto para Wushoue, et al.,(2014), a classe de antimicrobianos mais utilizados foram as cefalosporinas que representaram $28,6 \%$ do consumo total, seguido de penicilinas (20\%), macrolídeos (17,4\%) e fluoroquinolonas (10,5\%) realizado em 2011 a 2015 . Considerando esses resultados semelhantes ao presente estudo observa se que o grupo das cefalosporinas, carbapenêmicos, glicopeptídeos e penicilinas foram as classes que se destacaram com DDD maior.

De modo geral, conforme mostra o gráfico anterior, os valores de DDD das classes de antimicrobianos entre os anos 2015 e 2016 apresentaram uma diminuição estatisticamente significativa, já entre 2016 e 2017 teve um aumento estatisticamente significativo. Para tanto, observou-se que o hospital apresentou uma tendência crescente no consumo de antimicrobianos durante o ano de 2017. Este mesmo aumento foi observado em um estudo realizado na China e justificado pelo número de internações no hospital que quase dobrou durante o período de estudo elevando o consumo de antimicrobianos, (Wushouer, et al., 2014).

Com relação ao aumento do número de internações, no hospital em estudo no segundo semestre de 2016 foram disponibilizados mais leitos na UTI e abertura da Unidade de Assistência de Alta Complexidade (UNACON). Desta forma, o nível de complexidade dos pacientes internados aumentou, Porém, com o aumento de internação em 2016, houve uma redução de DDD e DOT quando comparado ao ano anterior, contudo pode ter contribuído para a elevação do consumo dos antimicrobianos. Assim, para paciente com alta complexidade a indicação do uso de antimicrobianos tanto profiláticos quanto para o controle de desenvolvimento de infecções oportunistas, principalmente por sepse de microrganismos Gram negativos, 
aumenta a administração de antimicrobianos de amplo espectro, consequentemente de alto custo, elevando os gastos no hospital (Odorizzi, et al 2017).

A utilização dos antimicrobianos, em termos econômicos, responde por um dos grupos em que os gastos hospitalares com medicamentos são maiores. Neste contexto, avaliações precisas dos custos com infecções adquiridas no hospital têm implicações importantes para os pacientes, gestores e pagadores Rodrigues, et al.,(2010). Portanto, realizou-se uma análise do consumo total em reais $(\mathrm{R} \$$ ) dos antimicrobianos no hospital universitário no período em estudo (gráfico 2).

Gráfico 2. Consumo total em Reais (R\$) dos antimicrobianos no período de 2015, 2016 e 2017 em um Hospital Universitário. Teresina-PI, 2018.

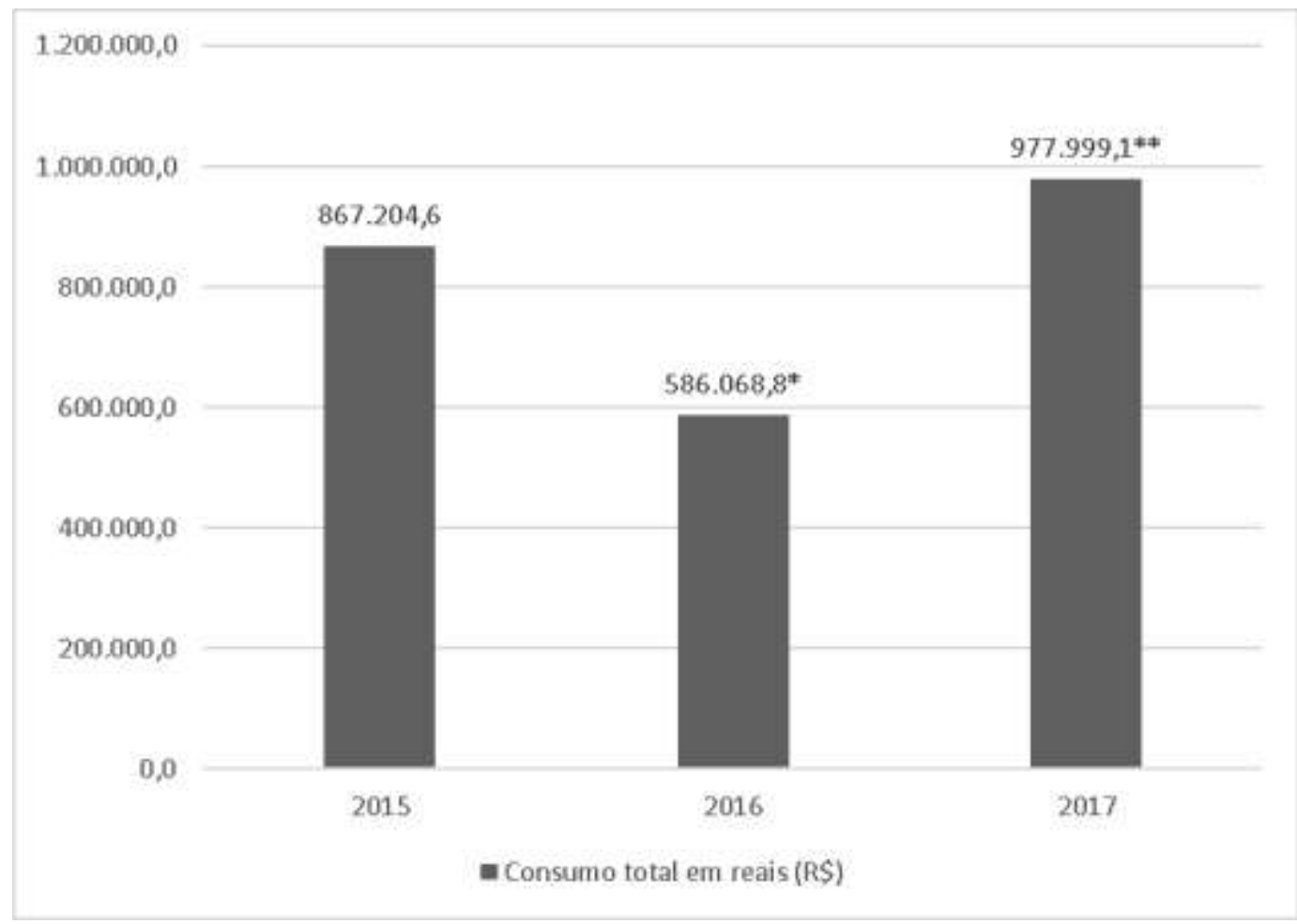

Legenda: A análise estatística foi baseada no teste t, que mostrou diferenças estatisticamente significativas para o consumo em reais $(\mathrm{R} \$$ ): Entre 2015 e 2016 observou uma diminuição estatisticamente significante ( $\mathrm{p}=0,02925)^{*}$, já entre 2016 e 2017 apresentou um aumento estatisticamente significante $(\mathrm{p}=0,004796) * *$. Fonte: Autores.

Portanto, entre os anos $2015(\mathrm{R} \$ 867.204,6)$ e $2016(\mathrm{R} \$ 586.068,8)$ o consumo total em reais ( $\mathrm{R} \$)$ dos antimicrobianos diminuiu com diferença estatisticamente significativa de ( $\mathrm{R} \$ 338,135,8)$, já entre 2016 e 2017 (R $977.999,1)$ notou-se um aumento neste consumo. Desta forma, algumas hipóteses podem ser levantadas para justificar o grande aumento evidenciado na despesa com agentes antimicrobianos, tais como: o aumento do consumo de agentes antimicrobianos no período em estudo, o aumento no número de pacientes internados, mudanças de preços, inflação ou diferenças entre compras e uso atual de medicamnetos (Guillot, et al., 2014) e (Costa, et al., 2013).

Entretanto, observou-se que durante os três anos de estudo as classes de antimicrobianos que representaram mais de $50 \%$ dos gastos para o hospital com antimicrobianos foram o grupo das penicilinas com $\mathrm{R} \$ 593.886,20$ (24,4\%), em seguida carbapenêmicos com R \$ 527.651,60 (21,7\%) e polimixinas com R\$277.213,00 (11,4\%).

No ambiente hospitalar, as unidades de terapia intensiva (UTI) em relação às outras unidades de internação são consideradas os epicentros de casos de infecções hospitalares, pois é onde se encontra o maior número de pacientes em estado 
crítico, em uso de procedimentos invasivos e com um grande arsenal terapêutico. Embora representem de 5 a $10 \%$ dos leitos de um hospital, estima-se que nelas ocorra cerca de 25\% das infecções hospitalares (Ibrahim et al., 2017).

Considerando isto, na tabela 02 , está disposto o consumo total em reais $(\mathrm{R} \$)$ dos postos de internações e Unidade de Terapia Intensiva (UTI) das classes de antimicrobianos em um hospital Universitário no período de 2015, 2016 e 2017.

Tabela 2. Consumo total em reais (R\$) por classes de antimicrobianos entre posto e UTI no período de 2015, 2016 e 2017 em um Hospital Universitário. Teresina-PI, 2018.

\begin{tabular}{lllllll}
\hline $\begin{array}{l}\text { Classes } \\
\text { antimicrobianos }\end{array}$ & dos & 2015 & & 2016 & & 2017 \\
& POSTO-RS & UTI-R $\$$ & POSTO-R\$ & UTI(R $\$)$ & POSTO-R\$ & UTI-R\$ \\
\hline Aminoglicosídeos & 886,1 & 204,9 & 853,8 & 328,5 & $1.623,7$ & 516,6 \\
Antifúngicos & $9.592,5$ & $3.908,9$ & $1.637,4$ & $18.211,2$ & $35.983,0$ & $37.465,7$ \\
Carbapenêmicos & $94.634,3$ & $55.339,4$ & $68.048,9$ & $54.510,5$ & $160.382,6$ & $94.736,0$ \\
Cefalosporinas & $49.785,3$ & $5.938,0$ & $51.316,0$ & $7.038,6$ & $50.815,2$ & $6.722,1$ \\
Glicilciclinas & $28.201,9$ & $18.901,3$ & $14.701,0$ & $12.600,8$ & $14.782,7$ & $18.020,3$ \\
Glicopeptídeo & $52.509,0$ & $18.050,2$ & $34.779,9$ & $17.374,6$ & $36.991,2$ & $37.277,7$ \\
Lincosamidas & $19.081,2$ & 426,3 & $9.704,9$ & 281,9 & $11.557,4$ & 547,2 \\
Macrolídeos & $25.538,2$ & $6.063,9$ & $16.520,1$ & $1.596,6$ & $13.319,3$ & $1.694,5$ \\
Nitroimidazóis & $12.229,9$ & 764,5 & $8.978,0$ & 899,4 & $13.809,7$ & $1.356,2$ \\
Oxazolidinonas & $33.977,5$ & $50.966,2$ & $16.541,7$ & $18.777,0$ & $9.210,7$ & $30.623,6$ \\
Penicilinas & $156.326,6$ & $31.298,3$ & $125.264,9$ & $40.849,1$ & $189.139,9$ & $51.007,4$ \\
Polimixinas & $34.655,9$ & $134.450,5$ & $18.745,3$ & $30.212,0$ & $15.243,8$ & $43.905,4$ \\
Quinolonas & $21.663,6$ & $1.810,3$ & $14.042,9$ & $2.253,6$ & $94.175,4$ & $7.092,0$ \\
\hline TOTAL & 539.082 & $328.122,5$ & $381.134,7$ & $204.933,8$ & $647.034,5$ & $330.964,7$ \\
\hline
\end{tabular}

Legenda: A análise estatística foi baseada no teste t para as três situações: Primeiro notou-se que os POSTOS tiveram maior custo estatisticamente significativas em relação a UTI durante os anos de 2015 ( $\mathrm{p}=0,01265), 2016$ ( $\mathrm{p}=0,04288)$ e 2017 ( $\mathrm{p}=0,04453$ ). Segundo para o POSTOS entre 2015 e 2016 apresentaram uma diminuição estatisticamente significante $(\mathrm{p}=0,0003302)^{*}$, já entre 2016 e 2017 observou um aumento estatisticamente significante $(\mathrm{p}=0,02905)^{* *}$. E em terceiro para a UTI teve um aumento estatisticamente significante a cada ano $(\mathrm{p}<0,05)$. Fonte: Autores.

Conforme expõe a tabela 02 , os antimicrobianos nos postos de internação tiveram maior consumo em reais (R\$) estatisticamente significativos em relação a UTI durante os anos de 2015, 2016 e 2017. Assim, os gastos com os antimicrobianos variam consideravelmente de hospital para hospital e em um mesmo local entre as diversas unidades de internação. Desta forma, para esse aumento dos gastos nos postos de internação, cabe ressaltar que a quantidade de pacientes internados é maior do que na UTI, levando ao seu alto custo.

Achado contrário a este estudo foi verificado por Onzi (2012), ao analisar os setores de internações do hospital, a UTI apresentou maiores gastos em relação às outras unidades. Entretanto, cabe ressaltar que o elevado consumo de antimicrobianos na UTI reflete a gravidade dos pacientes e apresentam as taxas de infecção mais elevadas nos hospitais, consequentemente aumento dos gastos.

Em vista disso, nos postos de internação entre os anos 2015 e 2016 notou-se que tiveram uma diminuição estatisticamente significativa, já entre 2016 e 2017 observou-se um aumento nos gastos com antimicrobianos. E na UTI, entre os anos houve um aumento estatisticamente significativo para o hospital. Dessa forma, para o aumento do consumo em reais 
tanto nos postos quanto na UTI dos antimicrobianos estudados, pode ser justificado pela abertura de leitos na UTI e UNACON com pacientes de alto grau de complexidade, como abordado anteriormente.

Desse modo, a complexidade o perfil de antimicrobianos utilizados na UTI se diferencia um pouco do restante das unidades hospitalares, visto a condição clínica do paciente. Sendo a principal diferença devido a unidade de terapia intensiva abrigarem pacientes de alto risco, fator relevante para o uso intenso de terapias antimicrobianas. Apesar dos esforços para minimizar esta utilização, muitos pacientes recebem antimicrobianos de amplo espectro, elevando bastante os custos para o hospital (Ibrahim et al., 2017).

$\mathrm{Na}$ grande maioria dos casos de infecções graves, é iniciada terapêutica empírica até que seja obtido o resultado das culturas com isolamento do agente causador da infecção. Ainda afirmam que o tratamento de escolha deve ser de amplo espectro, a fim de cobrir todos os possíveis microrganismos causadores, sendo modificado assim que haja a definição do agente etiológico e da sua sensibilidade aos antibióticos. Entretanto, a manutenção da terapia de amplo espectro é prejudicial por diversos fatores: possibilita maior toxicidade ao paciente em relação aos antibióticos de menor espectro, facilita as interações medicamentosas, encarece o tratamento e favorece a superinfecção por microrganismos resistentes (Sousa, et al,. 2014).

No entanto, deve ser reconhecido que o uso de antimicrobianos é complexo e que as métricas de consumo servem como um dos muitos indicadores potenciais de uso excessivo ou subutilização. Assim, a análise robusta de práticas adequadas do seu uso no nível hospitalar pode ser um alvo mais fácil e mais relevante para programas de administração no curto prazo. Como é o caso da avaliação entre a administração dos antimicrobianos nas apresentações parenterais por vias orais Scheetz, et al., (2016).

Diante disso, o presente estudo analisou o consumo em reais ( $\mathrm{R} \$$ ) dos antimicrobianos padronizados no Hospital Universitário que tinham as duas apresentações: parenterais e orais, no período de 2015, 2016 e 2017 (Tabela 3).

Tabela 3. Consumo em reais $(\mathrm{R} \$)$ dos antimicrobianos nas apresentações parentais e orais no período de 2015,2016 e

\begin{tabular}{crrrrrr}
\hline \multirow{2}{*}{ Antimicrobianos } & \multicolumn{2}{c}{2015} & \multicolumn{2}{c}{2016} & \multicolumn{2}{c}{2017} \\
& \multicolumn{1}{c}{ IV } & \multicolumn{1}{c}{ VO } & \multicolumn{1}{c}{ IV } & \multicolumn{1}{c}{ IV } & \multicolumn{1}{c}{ VO } \\
\hline Azitromicina & $19.643,0$ & 110,2 & $10.670,3$ & 46,8 & $8.280,7$ & 140,5 \\
Ciprofloxacino & $20.618,5$ & 258,1 & $13.469,3$ & 224,7 & $96.659,6$ & 555,6 \\
Claritromicina & $11.485,7$ & 363,1 & 7134,0 & 265,6 & $5.779,2$ & 813,4 \\
Clindamicina & $19.495,8$ & 11,7 & $9.986,8$ & 0,0 & $12.104,6$ & 0,0 \\
Fluconazol & $2.979,3$ & 17,4 & $2.379,7$ & 34,4 & $2.826,8$ & 54,2 \\
Levofloxacino & $2.182,2$ & 415,1 & $2.440,0$ & 162,5 & $3.907,0$ & 145,2 \\
Metronidazol & $11.136,5$ & $1.857,9$ & $8.410,4$ & $1.467,0$ & $14.544,8$ & 621,1 \\
\hline TOTAL & $87.541,0$ & $3.033,5$ & $54.490,4 *$ & $2.200,9 * \mathrm{a}$ & $144.102,7 * *$ & $2.329,9 * \mathrm{~b}$ \\
\hline
\end{tabular}

Legenda: A análise estatística foi baseada no teste t para as três situações: Primeiro, observou-se que os antimicrobianos por vias parenterais tiveram diferenças estatisticamente significativas das vias orais durante os anos 2015, 2016 e 2017 ( $p<0,05$ ). Segundo para a via parenteral entre 2015 e 2016 apresentaram uma diminuição estatisticamente significativa $(\mathrm{p}=0,009579)^{*}$, já entre 2016 e 2017 observou um aumento estatisticamente significativo ( $\mathrm{p}=0,01594)^{* *}$. E em terceiro para as vias orais entre 2015 e 2016 apresentaram uma diminuição estatisticamente significativa ( $\mathrm{p}=0,03937) * a$, já entre 2016 e 2017 observou um aumento estatisticamente significativo $(\mathrm{p}=0,05329) * \mathrm{~b}$. Fonte: Autores.

É possível observar que o consumo dos antimicrobianos por vias parenterais apresentaram diferenças estatisticamente significativas das vias orais durante os anos de 2015, 2016 e 2017. Dados semelhantes foram encontrados por Wushouer, et al., (2017), em seu estudo com os antimicrobianos parenterais que representaram $40 \%$ do consumo total e $87 \%$ das despesas totais 
de medicamentos nos anos de 2011 a 2015. Uma vez que as apresentações parenterais são mais caras do que as de vias orais, isso poderia contribuir para uma distorção na seleção da via de administração.

Entretanto, o uso excessivo de apresentações parenterais quando as formulações orais seriam mais apropriadas, é um dos fatores chave para o uso irracional de medicamentos. Assim como, as mudanças dessas vias diminuem o tempo de permanência dos pacientes internados, bem como os gastos para o hospital. Além disso, uma vez que os relatórios de cultura e sensibilidade estão disponíveis, permite selecionar um antimicrobiano via oral mais barato, que é tão eficaz quanto por via parenteral (Apolinário et al., 2017).

Uma nova abordagem para troca dependendo do estado clínico do paciente seria a terapia sequencial que é o termo empregado para designar a conversão do tratamento antimicrobiano por via endovenosa para via oral, empregando o mesmo fármaco. Se a conversão ocorrer empregando-se outro fármaco da mesma classe farmacológica, denomina-se terapia de transferência (Therapy switch), Costa, et al.,2014. Assim, em um estudo realizado no hospital no sul da Índia por um período de 6 meses mostrou que o custo médio dos antimicrobianos e o tempo de permanência dos pacientes poderiam ser reduzidos devido à mudança precoce da terapia parenteral para a oral Cyriace, J.M \& James E. (2014).

Além disso, conforme mostra a tabela acima, os antimicrobianos que demonstraram um aumento durante o período em estudo nas apresentações por via parenteral foram ciprofloxacino $(\mathrm{R}$ \$ 20.618,5 a $\mathrm{R} \$ 96.659,6)$ e metronidazol ( $\mathrm{R} \$ 11.136,5$ a $\mathrm{R} \$ 14.544,8)$, levofloxacino ( $\mathrm{R}$ \$2.182,2 a $\mathrm{R} \$ 3.907,0)$. Assim, uma das possibilidades para a diminuição dos gastos com esses antimicrobianos, dependendo das condições clínicas dos pacientes, seria a permuta pelas apresentações de via orais, gerando custos menores ao hospital.

O hospital universitário, durante o período em estudo possuía em seu elenco apenas sete antimicrobianos padronizados nas apresentações de via oral. Assim, de modo geral, pode ser observado que o consumo em reais para os antimicrobianos com apresentações via oral entre os anos 2015 e 2016 apresentou uma diminuição estatisticamente significativa, já entre os anos 2016 e 2017 apresentaram um aumento estatisticamente significativo. Assim, os antimicrobianos que apresentaram uma diminuição nos gastos foram a clindamicina ( $R$ \$ 11,7 a $R$ \$ 0,0), levofloxacino ( $R \$ 415,1$ a $R \$ 145,2)$ e metronidazol ( $\mathrm{R} \$ 1.857,9$ a $\mathrm{R} \$ 621,1)$. É importante esclarecer que o custo zero da clindamicina deve-se a retirada da padronização em 2015. Enquanto para levofloxacino e metronidazol, observou-se desabastecimento desses itens. A utilização das apresentações por via oral deve ser estimulada, pois o seu uso racional são formas mais econômicos e diminuem o tempo de hospitalização.

\section{Conclusão}

$\mathrm{O}$ aumento do consumo dos antimicrobianos geraram maiores gastos para o hospital ao longo dos anos, sendo um dos fatores o aumento do número de pacientes com alta complexidade, que necessitam de terapias medicamentosas mais complexas e consequentemente mais caras. Além disso, percebe-se a necessidade de mais estudos de utilização de antimicrobianos, uma vez que esses medicamentos são essenciais para o tratamento das doenças infecciosas e têm uma influência incontestável na diversidade microbiana, com o impacto na resistência dos microrganismos sendo o seu uso inadequado nocivo para todos.

Desta forma, conclui-se que os conhecimentos do monitoramento de consumo dos medicamentos fornecem argumentos que norteiam para o gerenciamento do uso racional dos antimicrobianos e contribuem para a tomada de decisões clínicas e gerenciais no ambiente hospitalar. 


\section{Referências}

Apolinário, Alexsandra Conceição (2017) Polimerossomos versus lipossomos: a evolução da "bala mágica”. Química Nova, 40(7), 810-817.

Barbosa, J. A. A., Belém, L. de F., Sette, I. M. F., Farias, T. de, Pereira, G. J. da S., \& Júnior, E. D. da S. (2009). Utilização de antimicrobianos em pacientes oncológicos hospitalizados. Rev. Bras. Farm. Marc 69-74.

Bell, B. G., Schellevis, F., Stobberingh, E., Goossen, H., \& Pringle, M. (2014). A systematic review and meta-analysis of the effects of antibiotic consumption on antibiotic resistance. BMC Infect. Dis; 9: 14:13.

Bitterman, R., Hussein, K., Leibovici, L., Carmeli, Y., \& Paul, M. (2016)Systematic review of antibiotic consumption in acute care hospitals. Clin Microbiol Infect. 22(6):561.e7-561.e19

Brasil. Ministério da Saúde. Norma técnica GVIMS/GGTES ANVISA Nº5/2017.

Cyriace, J. M., \& James, E. (2014) Switch over from intravenous to oral therapy: A concise overview. J. Pharmacol Pharmacother. 5(2):83-7

Costa, J..M., Abelha, L..L., Pinto, I. V. L., \& Reis, A. M. M. (2014) Análise de um programa para otimização da transição do cuidado de pacientes em terapia antimicrobiana. Rev. APS.

Costa, R. S., Gabetto, R. S. de S., \& Caribé, R. A. (2013). Análise farmacoeconômica de minimização de custos para protocolo de antibioticoterapia para pneumonia adquirida em comunidade de um hospital estadual do Rio de Janeiro/RJ. Rev. Bras. Farm. Hosp. Serv. Saúde.

Dubrovskaya, Y., Scipione, M. R., Siegfried, J., Jen, S. P., Pham, V., Papadopoulos, J., Decano, A., Lewis, T., \& Dabestani, A. (2017) Multilayer Model of Pharmacy Participation in the Antimicrobial Stewardship Program at a Large Academic Medical Center. Hosp Pharm. 52(9):628-634.

Fernandes, I. de Q., Sousa, H. F. de, Brito, M. A. M., Tavares, S. N., Matos, V. C. de \& Souza, M. de O. B (2012). Impacto farmacoeconômico da racionalização do uso de antimicrobianos em unidades de terapia intensiva. Rev Bras Farm Hosp Serv Saúde.

Grosbelli Vicari, N., Gonçalves de Oliveira, A., Ferrazzoli Devienne Vicentine, K., \& Dias Paiva, A. (2020). Atividade Antimicrobiana Do Extrato De Casearia sylvestris CONTRA Enterococcus faecium Resistentes À Vancomicina. Revista Multidisciplinar Em Saúde, 1 (2), 46.

Guillot, J., \& Lebel, D. (2014). Usefulness of Defined Daily Dose and Days of Therapy in Pediatrics and Obstetrics-Gynecology: A Comparative Analysis of Antifungal Drugs (2000-2001, 2005-2006, and 2010-2011). J Pediatr Pharmacol Ther; 19(3):196-201.

Ibrahim, Mohammed, K. (2017). Design, synthesis, molecular modeling and antihyperglycemic evaluation of novel quinoxaline derivatives as potential PPAR $\gamma$ and SUR agonists. Bioorganic \& Medicinal Chemistry, 25(4), 1496-1513.

Lobo, L. G., Ramos, F., Moretti, M. M., \& Alves, P. H. (2017). Resultados de um Programa Multidisciplinar de Otimização do Uso de Antimicrobianos. R Epidemiol Control Infec; 7(1):47-51.

Momattin, H., Al-alib, A. Y., Mohammeda, K., Al-tawfiqc, J. (2018). Benchmarking of antibiotic usage: An adjustment to reflect antibioticstewardship program outcome in a hospital in Saudi Arabia. J Infect Public Health. 11(3):310-313.

Onzi, P. de S., Hoffman, S. P., \& Camargo, A. L. (2011). Avaliação do consumo de antimicrobianos injetáveis de um hospital privado no ano de 2009. R. Bras. Farm. Hosp. Serv. Saúde 2(2):20-25.

Odorizzi, V. F. et al. (2017). Portadores assintomáticos de Staphylococcus aureus na cavidade nasal entre indígenas da etnia xerente, Tocantínia, Tocantins, Brasil. Anais do Seminário Científico do UNIFACIG, n. 2.

Polk, R. E., et al. (2007). Measurement of Adult Antibacterial Drug Use in 130 US Hospitals: Comparison of Defined Daily Dose and Days of Therapy. Clinical Infectious Diseases; 44(5):664-70

Rodrigues, F. A., \& Bestoldi, A. D. (2010). Perfil da utilização de antimicrobianos em um hospital privado. Ciênc Saúde Coletiva; $15: 1239-1247$.

Santos Júnior, J. G. A. (2021). Desenvolvimento, caracterização e avaliação in vitro da atividade antibacteriana e formulações lipossomais. 73 f. Tese (Doutorado em Desenvolvimento e Inovação Tecnológica em Medicamentos) - Faculdade de Farmácia, Odontologia e Enfermagem, Universidade Federal do Ceará, Fortaleza, 2021.

Siegfried, J., Merchan, C., Scipione, M. R., Papadopoulos, J., Dabestani, A., \& Dubrovskaya, Y. (2017). Role of postgraduate year 2 pharmacy residents in providing weekend antimicrobial stewardship coverage in an academic medical center. Am J Health Syst Pharm. 74(6):417-423

Sous, A. D. C. P., et al. (2014). Estudo da utilização do meropenem no hospital universitário alcides carneiro - HUAC. In: Congresso Brasileiro de Ciências da Saúde.

Scheetz, M. H., et al. (2016). Investigating the Extremes of Antibiotic Use with an Epidemiologic Framework. Antimicrob Agents Chemother; 60(6):3265-9

World Health Organization - WHO.(2012). The evolving threat of antimicrobial resistance options for action.

Wushouer, H., et al. (2017).Trends and patterns of antibiotic consumption in China's tertiary hospitals: Based on a 5 year surveillance with sales records, 20112015. PLOS ONE.

Vasconcelos, D. V., et al. (2015). O uso de antimicrobianos no âmbito hospitalar e as atribuições do farmacêutico na Comissão de Controle Infecção Hospitalar (CCIH). Rev Elet de Ciênc Hum, Saúde e Tecn.; 8(2). 